\section{(A) OPEN ACCESS}

\title{
Identification of the $N F-\kappa B$ activating protein-like locus as a risk locus for rheumatoid arthritis
}

\author{
Gang Xie, ${ }^{1,3}$ Yue Lu, ${ }^{2}$ Ye Sun, ${ }^{1,3}$ Steven Shiyang Zhang, ${ }^{1}$ Edward Clark Keystone, ${ }^{3}$ \\ Peter K Gregersen, ${ }^{4}$ Robert M Plenge, ${ }^{5}$ Christopher I Amos, ${ }^{2}$ \\ Katherine A Siminovitch ${ }^{1,3,6}$
}

\begin{abstract}
- Additional data are published online only. To view these files please visit the journal online (http://dx.doi.org/ 10.1136/annrheumdis-2012202076).

${ }^{1}$ Mount Sinai Hospital Samuel Lunenfeld Research Institute and Toronto General Research Institute, Toronto, Ontario, Canada

${ }^{2}$ Department of Epidemiology, University of Texas M.D.

Anderson Cancer Center, Houston, Texas, USA

${ }^{3}$ Rebecca MacDonald Centre for Arthritis, Department of

Medicine, Mount Sinai

Hospital, University of Toronto, Toronto, Ontario, Canada

${ }^{4}$ Robert S. Boas Center for Genomics and Human

Genetics, The Feinstein Institute for Medical Research, North Shore-Long Island Jewish Health System, Manhasset, New York, USA ${ }^{5}$ Division of Rheumatology, Harvard Medical School, Brigham and Women's Hospital, Boston,

Massachusetts, USA

${ }^{6}$ Departments of Immunology and Molecular Genetics, University of Toronto, Toronto, Ontario, Canada
\end{abstract}

\section{Correspondence to}

Dr Katherine A Siminovitch, Mount Sinai Hospital, Lunenfeld Research Institute and Toronto General Research Institute, 600 University Ave, Room 778D, Toronto, Ontario,

Canada M5G 1X5;

ksimin@mshri.on.ca

Received 23 May 2012 Accepted 21 October 2012 Published Online First 6 December 2012

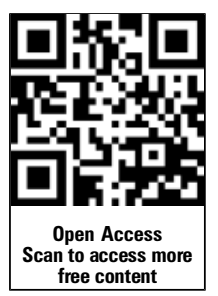

\section{ABSTRACT}

Objective To fine-map the $N F-\kappa B$ activating protein-like (NKAPL) locus identified in a prior genome-wide study as a possible rheumatoid arthritis (RA) risk locus and thereby delineate additional variants with stronger and/or independent disease association.

Methods Genotypes for 101 SNPs across the NKAPL locus on chromosome 6p22.1 were obtained on 1368 Canadian RA cases and 1471 controls. Single marker associations were examined using logistic regression and the most strongly associated NKAPL locus SNPs then typed in another Canadian and a US-based RA case/ control cohort.

Results Fine-mapping analyses identified six NKAPL locus variants in a single haplotype block showing association with $p \leq 5.6 \times 10^{-8}$ in the combined Canadian cohort. Among these SNPs, rs35656932 in the zinc finger 193 gene and rs13208096 in the NKAPL gene remained significant after conditional logistic regression, contributed independently to risk for disease, and were replicated in the US cohort $\left(P_{\text {comb }}=4.24 \times 10^{-10}\right.$ and $2.44 \times 10^{-9}$, respectively). These associations remained significant after conditioning on SNPs tagging the HLAshared epitope (SE) DRB1*0401 allele and were significantly stronger in the HLA-SE negative versus positive subgroup, with a significant negative interaction apparent between HLA-DRB1 SE and NKAPL risk alleles. Conclusions By illuminating additional NKAPL variants with highly significant effects on risk that are distinct from, but interactive with those arising from the HLADRB1 locus, our data conclusively identify NKAPL as an RA susceptibility locus.

\section{INTRODUCTION}

Rheumatoid arthritis (RA) is a chronic autoimmune disease primarily associated with inflammation of the synovial joints and affecting up to $1 \%$ of the population worldwide. Although the complex interplay of genetic and environmental factors underpinning RA are not well understood, major inroads have been made in mapping gene loci associated with risk for this disease. ${ }^{1}$ In addition to the $H L A-D R B$ locus, over 35 non-major histocompatibility complex (MHC) RA risk loci have emerged from genome-wide association studies (GWAS) and subsequent GWAS meta-analyses of the GWAS datasets. ${ }^{2-10}$

Through a genome-wide scan of 2418 RA patients and 4504 healthy controls ascertained in Canada and the USA, we previously identified an association of
RA with the REL NF- $\kappa B$ transcription factor locus and also confirmed already identified disease associations with the PTPN22, CTLA4, TNFAIP3, BLK and TRAF1/C5 genes. ${ }^{9}$ Our data also showed strongly suggestive signals ( $\mathrm{P}_{\text {GWAS }}$ values between $8.2 \times 10^{-7}$ and $5.28 \times 10^{-8}$ ) emanating from a cluster of Single nucleotide polymorphisms (SNP) across a $70 \mathrm{~kb}$ region on chromosome $6 \mathrm{p} 22.1$ encompassing the $N F-\kappa B$ activating protein-like gene (NKAPL) as well as three Zinc finger protein transcription factors ZNF193, ZNF307 and ZNF187. A follow-up GWAS meta-analysis of $5505 \mathrm{RA}$ and 22603 controls of European descent ${ }^{10}$ also revealed 13 genotyped or imputed SNPs across a $150 \mathrm{~kb}$ region encompassing the NKAPL locus to be strongly associated ( $\mathrm{P}_{\text {meta }}$ between $1 \times 10^{-10}$ and $1 \times 10^{-13}$ ) with RA (R Plenge, personal communication). Although NKAPL functions are unknown, its protein product shows $90 \%$ sequence similarity to NF- $\mathrm{KB}$ activating protein (NKAP), a protein implicated in $N F-\kappa B$-mediated transcriptional activation of TNF and IL-1. ${ }^{11}$ As data from our group and others have also implicated other genes from the $N F-\kappa B$ signalling pathway (eg, REL, CD40, TRAF1, TNFAIP3, PRKCQ and TNFRSF14) in RA susceptibility, ${ }^{9} 1213$ the NKAPL gene represents a compelling potential candidate gene for RA. We therefore undertook fine-mapping studies of the NKAPL locus aimed at confirming this association, identifying those variants providing the strongest association signal and defining whether such variants act together or independently of one another and/or the HLA-DRB1 locus in conferring risk for RA.

\section{METHODS}

\section{Subjects}

Study cohorts (see online supplementary methods) include: 3979 subjects of European origin (2078 RA patients and 1901 healthy controls) recruited independently from two clinical centres in Canada, Toronto (1368 cases and 1471 healthy controls) and Halifax (710 cases and 430 healthy controls) and a third cohort including 2064 subjects of European ancestry ascertained in the USA as part of the Brigham Rheumatoid Arthritis Sequential Study and used here for replication analysis.

\section{SNP selection}

SNPs from a $372 \mathrm{~kb}$ interval across the NKAPL locus on chromosome $6 \mathrm{p} 22.1$ were selected primarily based on at least one of the following criteria: 
(1) HapMap phase III data identifying the SNP as a tag SNP with minor allele frequency $>0.01$ and $r^{2}$ threshold of 0.8 or (2) localisation within $150 \mathrm{~kb}$ upstream or downstream of SNPs most significantly associated with RA in our GWAS. Other SNPs studied were: the autoimmune disease-associated PTPN22 rs2476601 ${ }^{14}$ and two SNPs (rs660895 and rs6910071) that tag the HLA-DRB1*0401 allele on chromosome $6 \mathrm{p} 21.3$. $^{15}$

\section{Statistical analyses}

Hardy-Weinberg equilibrium, allelic association and conditional logistic regression analyses were performed using PLINK software V1.07 (http://pngu.mgh.harvard.edu/purcell/plink/). For the allelic association tests, the threshold for declaring significance was assigned according to Benjamini and Hochberg's False Discovery Rate method and set at $p<5.00 \times 10^{-4}(0.05 / 101)$. Cochran-Mantel Haenszel $\chi^{2}$ analysis was used to combine $p$ values and calculate OR from the Canadian and US cohorts and an R-script (http://www.rproject.org/) was used to generate figures. Haplotype block structure, depicted using Haploview software V4.1 (http://www.broad.mit.edu/mpg/haploview), was defined according to the criteria established by Gabriel ${ }^{16}$ and the pairwise estimates of standardised Lewontin's disequilibrium coefficient $\left(\mathrm{D}^{\prime}\right)$, whereas the linkage disequilibrium (LD) among pairs of SNPs was characterised according to the square of the correlation coefficient $\left(\mathrm{r}^{2}\right)$. Conditional logistic regression analyses of multiple markers were performed using SAS V9.13 (SAS Institute Inc., Cary, North Carolina, USA). Gene-gene interaction analysis was performed by case-only interaction analysis in which a logistic regression model was used to test for an association of shared epitope (SE) positivity with NKAPL risk alleles (coded in an additive fashion as $-1,0$ or 1 for no, 1 or 2 risk alleles, respectively). For multinomial logistic regression modelling, ${ }^{17}$ controls were considered as the lowest risk outcome, SE negative cases as the intermediate risk outcome and SE positive cases as the highest risk outcome, and these multiple outcomes were then assessed according to number of NKAPL risk alleles. The statistical power for this study was evaluated using CaTS software (http://www.sph.umich.edu/ csg/abecasis/CaTS/) with the following parameters: disease prevalence 0.01 , disease allele frequency $0.2, \alpha=0.0005$ (0.05/ 101). Power to detect associations with relative risk of 1.5 was estimated to be $99.4 \%$.

\section{RESULTS}

Fine-mapping of the RA-associated NKAPL locus at 6p22.1

To identify risk allele(s) at the NKAPL locus, we genotyped 1368 RA cases and 1471 controls from Toronto for 105 SNPs across a $372 \mathrm{~kb}$ genomic region encompassing the NKAPL gene. Characteristics of the study design and subjects are outlined in online supplementary figure S1. Among the 101 SNPs that passed quality control, 16 achieved the set significance threshold of $p<5.00 \times 10^{-4}$ with the top six markers showing associations with disease $\left(p<6.00 \times 10^{-7}\right)$ that remained highly significant ( $p$ values $1.80 \times 10^{-6}-8.60 \times 10^{-6}$ ) after False Discovery Rate correction (table 1 and online supplementary table S1). Haploview analysis of pairwise LD among the 101 SNPs revealed that these six most strongly associated SNPs map within a $70 \mathrm{~kb}$ segment representing the middle of three haplotype blocks across this region and containing the NKAPL gene and three zinc finger transcription factor genes, ZNF193, ZNF307 and ZNF187 (figure 1 and see online supplementary figure $\mathrm{S} 2$ ). The strongest association signal $\left(p=2.48 \times 10^{-8}\right)$ came from a ZNF187 intronic SNP (rs67998226) at the distal end of this haplotype block, but these variants were all in strong $\mathrm{LD}$ with one another, the pairwise $\mathrm{LD}$

Table 1 List of the six NKAPL locus SNPs showing the most significant association with rheumatoid arthritis in the Toronto- and Halifax-based case/control cohorts.

\begin{tabular}{|c|c|c|c|c|c|c|c|c|c|}
\hline \multirow[b]{2}{*}{ SNP } & \multirow[b]{2}{*}{ Gene } & \multirow[b]{2}{*}{ Risk allele } & \multirow[b]{2}{*}{ Sample cohort } & \multicolumn{2}{|l|}{ RAF } & \multirow[b]{2}{*}{ OR (95\% Cl) } & \multirow[b]{2}{*}{ p Value ${ }^{*}$} & \multirow[b]{2}{*}{$P_{\text {FDR }}$ value } & \multirow[b]{2}{*}{ Cochran $0 \mathrm{p}$ value } \\
\hline & & & & Case & Control & & & & \\
\hline \multirow[t]{2}{*}{ rs 13195291} & ZNF193 & A & Toronto & 0.115 & 0.075 & 1.61 (1.33 to 1.94$)$ & $5.03 \times 10^{-7}$ & $8.60 \times 10^{-6}$ & \\
\hline & & & Combinedt & 0.116 & 0.077 & 1.56 (1.33 to 1.82 ) & $2.79 \times 10^{-8}$ & $4.20 \times 10^{-8}$ & 0.45 \\
\hline \multirow[t]{2}{*}{ rs 35656932} & ZNF193 & $\mathrm{T}$ & Toronto & 0.118 & 0.075 & 1.66 (1.38 to 1.99$)$ & $3.82 \times 10^{-8}$ & $1.80 \times 10^{-6}$ & \\
\hline & & & Halifax & 0.119 & 0.083 & 1.48 (1.11 to 1.99$)$ & $8.31 \times 10^{-3}$ & 0.02 & \\
\hline & & & Halifax & 0.11 & 0.088 & 1.29 (0.96 to 1.74$)$ & 0.09 & 0.10 & \\
\hline & & & Combined & 0.114 & 0.078 & 1.53 (1.31 to 1.79$)$ & $5.66 \times 10^{-8}$ & $5.60 \times 10^{-8}$ & 0.20 \\
\hline \multirow[t]{3}{*}{ rs17720293 } & ZNF307 & $\mathrm{T}$ & Toronto & 0.135 & 0.089 & $1.60(1.35$ to 1.90$)$ & $5.27 \times 10^{-8}$ & $1.80 \times 10^{-6}$ & \\
\hline & & & Halifax & 0.129 & 0.107 & $1.23(0.94$ to 1.60$)$ & 0.139 & 0.14 & \\
\hline & & & Combined & 0.133 & 0.093 & 1.49 (1.29 to 1.72$)$ & $4.29 \times 10^{-8}$ & $5.10 \times 10^{-8}$ & 0.10 \\
\hline & & & Halifax & 0.122 & 0.086 & 1.47 (1.10 to 1.97$)$ & $9.88 \times 10^{-3}$ & 0.02 & \\
\hline & & & Combined & 0.122 & 0.079 & 1.62 (1.39 to 1.88$)$ & $6.53 \times 10^{-10}$ & $2.20 \times 10^{-9}$ & 0.48 \\
\hline
\end{tabular}

Genotype data are shown for six SNPs tested in 1368 cases and 1471 controls from Toronto in step 1 and replication in 710 cases and 430 controls from Halifax in step 2 . Data for all other SNPs tested in Toronto cohort are shown in online supplementary table S1.

${ }^{*}$ Nominal $p$ value from an allele-based case-control comparison with 1 degree of freedom; $p<0.05$ are highlighted in bold type. $P_{F D R}$ corresponds to $p$ values adjusted for multiple testing using the False Discovery Method implemented in PLINK.

†Genotyping data from the Toronto and Halifax cohorts were merged in PLINK and combined $\mathrm{P}_{\text {Can }}$ values for association evaluated using Cochran-Mantel Haenszel tests and the Cochran 0 test for heterogeneity.

FDR, False Discovery Rate; NKAPL, NF-kB activating protein-like; RAF, risk allele frequency. 


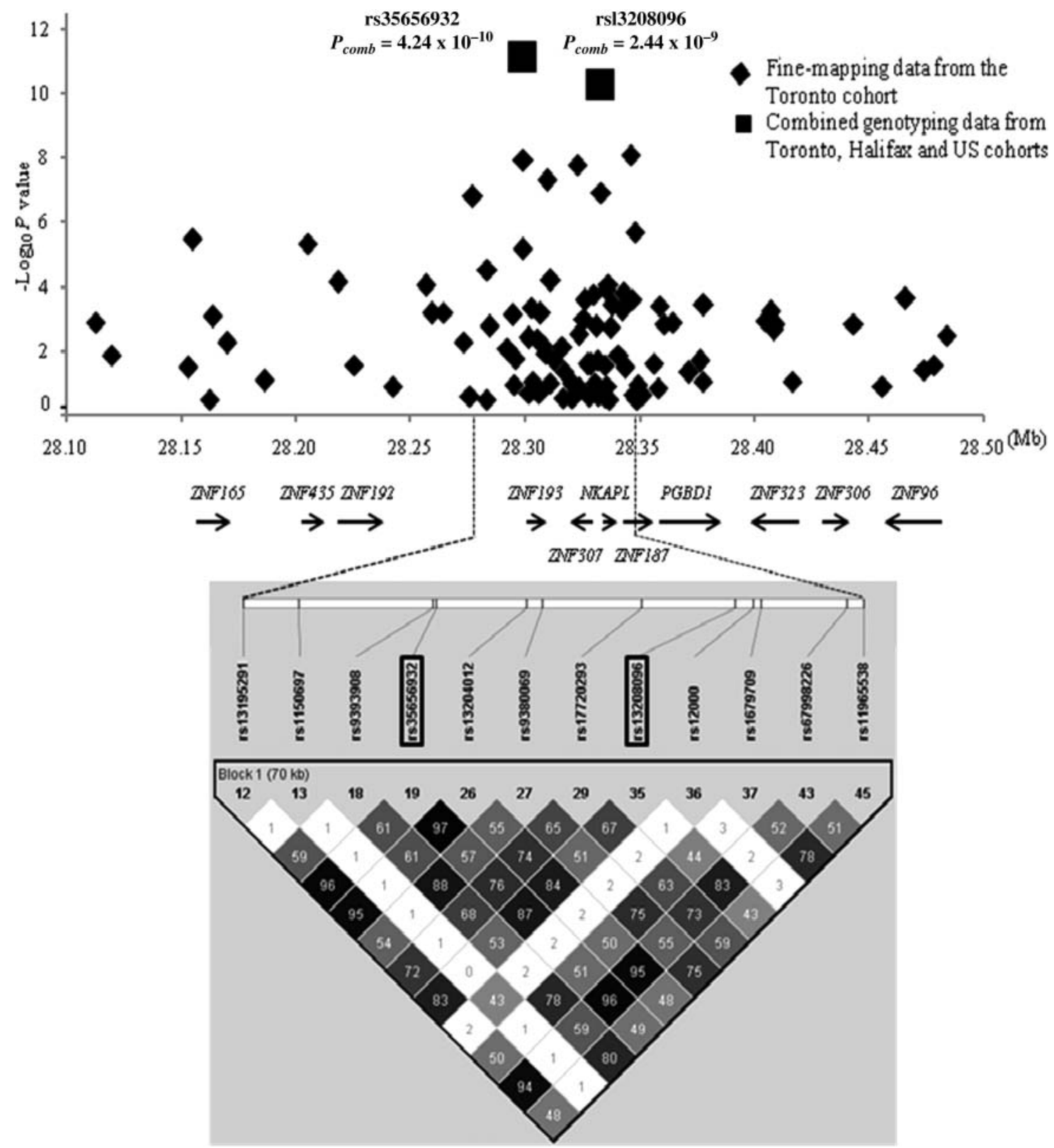

Figure 1 Case-control association results of SNPs (single nucleotide polymorphisms) at the NF-kB activating protein-like (NKAPL) locus on 6p22.1. SNPs genotyped in the fine mapping $(\diamond)$ analysis of the Toronto-based case/control cohort. Results of combined analyses for the rs35656932 and rs13208096 are shown (घ). Positions of the genes across the NKAPL locus are shown in the middle panel. The lower panel shows the linkage disequilibrium relationships between 12 of the most strongly associated SNPs $\left(p<5.0 \times 10^{-4}\right)$ across an $\sim 70$ kb region encompassing the ZNF193, ZNF307, NKAPL and ZNF187 genes. The numbers shown in the squares in the lower panel are $r^{2}$ values representing the correlation coefficient for a given marker pair. The shade intensity lightens in parallel with diminishing correlation coefficient $\left(r^{2}\right)$ values $\left(r^{2}=1:\right.$ black, $r^{2}=0:$ white $)$.

$\left(\mathrm{r}^{2}\right)$ between rs67998226 and either the three SNPs across the more proximal ZNF193 gene (rs13195291, rs35656932 and rs13204012) or the NKAPL promoter region SNP (rs13208096) being $>0.94$ and 0.83 , respectively, and between rs35656932 and rs13208096 being 0.87 .

To further examine effects of this locus on RA susceptibility, the six most significant SNPs were also typed in a Halifax-derived cohort including 710 RA patients and 430 controls. Four of the six associations (rs13195291, rs35656932, rs13208096 and rs67998226) were replicated in this cohort, with combined (Toronto and Halifax) association signals $\left(\mathrm{P}_{\text {CAN }}\right)$ ranging from $5.60 \times 10^{-8}$ to $2.22 \times 10^{-9}$ (table 1 ).

The extent to which the signals observed in the combined analysis were independent of one another was next examined using stepwise logistic regression analysis wherein variables were iteratively added into an empty model. This analysis identified the ZNF193/NKAPL locus rs35656932 SNP, which is in strong LD $\left(\mathrm{r}^{2}=0.96\right)$ with the ZNF187 rs67998226 SNP as the variant most strongly associated with risk for RA, but also suggested that both NKAPL rs13208096 and rs3656932 SNP alleles influence risk for RA (table 2). Additional conditional analyses of the six SNPs revealed that both rs35656932 and rs13208096 remain significantly associated with disease after conditioning on each marker (see online supplementary table S2). Because these data suggest independent contributions of alleles of rs35656932 and rs13208096 on risk, associations of these two variants with disease were next explored in a third (US) cohort including 863 cases and 1201 controls. The two SNPs both 
Table 2 Stepwise logistic regression in the two SNP models

\begin{tabular}{lll}
\hline SNP added to the model & p Value & OR (95\% Cl) \\
\hline rs35656932 & 0.003 & $5.82(1.82$ to 18.58$)$ \\
rs13208096 & 0.030 & $3.57(1.12$ to 11.11$)$ \\
\hline
\end{tabular}

Stepwise logistic regression results based on analysis of the six most strongly associated NKAPL locus SNPs in the 2078 RA cases and 1901 controls from Canada.

replicated in this cohort (table 3): combined analyses of all 2941 cases and 3102 controls typed for these SNPs (table 3) yielding highly significant $\mathrm{P}_{\text {comb values }}\left(4.24 \times 10^{-10}\right.$ for rs35656932 and $2.44 \times 10^{-9}$ for rs13208096).

\section{Variants of the NKAPL locus and the HLA region jointly} contribute to risk for RA

Because the NKAPL locus maps to chromosome 6p22.1, evaluation of its disease association may be confounded by effects of the HLA-DRB1 locus at 6p21.3, a locus which encodes the RA-predisposing SE, confers much of the genetic risk for RA, and maps in a region of extensive $\mathrm{LD}^{15}{ }^{18-23}$ Although the NKAPL gene lies $4306 \mathrm{~kb}$ upstream of the RA risk-related HLA-DRB 1 gene, the extent to which its association with RA may reflect $\mathrm{LD}$ with $H L A-D R B 1$ risk alleles was investigated by genotyping the Canadian cases (2078) and controls (1901) for two SNPs (rs660895 and rs6910071) that tag one of the most common SE encoding alleles, $H L A-D R B 1^{*} 0401$, reassessing the NKAPL-disease association by logistic regression analysis with conditioning on the HLA-DRB $1^{*} 0401$ SNPs. This analysis confirmed the strong association of RA with both tag SNPs, the signals reaching $p=2.04 \times 10^{-75}$ and $p=2.39 \times 10^{-63}$ for rs660895 and rs6910071, respectively (see online supplementary table S3). Importantly, the association signals from each of the six NKAPL locus SNPs remained highly significant $\left(p<1 \times 10^{-10}\right)$ after conditioning on the DRB1 tag SNPs (see online supplementary table S4). Analyses of the pairwise LD between the two tag SNPs and each of the 55 SNPs with nominal evidence $(p<0.05)$ for disease association in the initial analysis of Toronto controls also revealed no significant $\mathrm{LD}\left(\mathrm{r}^{2}<0.01\right.$ for each SNP pair) between any of these SNPs and the HLA-DRB1 tag SNPs (data not shown). These findings imply that the association signal at the NKAPL locus represents an effect on risk that is not attributable to LD with the HLA-DRB1*0401 allele.

To further evaluate the relationship between NKAPL and HLA locus effects on risk, we additionally used data from a large panel of British subjects genotyped for HLA alleles to impute, in the subset of the Canadian cohort included in our previous GWAS, ${ }^{9} 137$ HLA alleles encoding classic HLA-A, B, C, DRB1,
$\mathrm{DOA}$ and $\mathrm{DOB}$ molecules. Association tests were performed in a dataset combining the imputed alleles, and the GWAS-derived HLA region as well as the fine mapping-derived SNP genotypes. Single SNP association tests performed using this combined dataset and assuming an additive model (implemented in PLINK software) identified 379 SNPs (data not shown) or HLA genotypes with $\mathrm{p}$ values less than $1.0 \times 10^{-4}$ (see online supplementary table S5). These variants together with the six candidate SNPs identified by fine mapping were subjected to stepwise logistic regression analysis using SAS and a p value set at $<0.01$ as the criterion to enter and remain in the model. From this analysis, seven SNPs (including rs35656932), but no classical HLA alleles were retained in the model, suggesting that risk for disease at this locus is better explained by effects of SNPs rather than HLA alleles (see online supplementary table S6A). Stepwise logistic regression was then repeated with $H L A-D R B 1^{*} 0401$ forced into the model. After this analysis, five variants, including HLA-DRB1*0401 ( $\mathrm{p}=0.04)$, were retained in the model, although this HLA allele did not reach our significance criterion of $p<0.01$ (see online supplementary table S6B). These data provide further evidence that NKAPL variant(s) per se contribute to risk for RA.

Relevance of HLA genotypes to the NKAPL locus effect on risk for RA was also explored by assessing the extent to which the NKAPL region rs35656932 and rs13208096 SNPs associate with disease in cases stratified based on presence or absence of the SE alleles or of anticyclic citrullinated peptide (anti-CCP) antibody, an autoantibody strongly associated with SE alleles. ${ }^{24}$ This analysis revealed the disease associations for both risk alleles to be much higher in the SE negative $\left(p=1.20 \times 10^{-12}\right.$ for rs35656932 and $3.50 \times 10^{-11}$ for rs13208096) than in the SE positive $\left(p=4.70 \times 10^{-5}\right.$ and $8.10 \times 10^{-5}$, respectively) subgroup (table 4). By contrast, the association signals from these loci were essentially the same in the anti-CCP positive and negative subsets. Because a disparity between SE and anti-CCP status effects on this association was not expected, the stratified subgroups were also genotyped for the PTPN22 gene rs2476601 SNP for which an association with RA susceptibility is well established and thought to correlate with SE and anti-CCP positivity. ${ }^{25-27}$ This analysis confirmed the strong association of RA with the rs 2476601 variant $\left(p=2.72 \times 10^{-15}\right.$; see online supplementary table S3) as well as the positive effects of the SE $\left(p=1.40 \times 10^{-16}\right.$ SE positive vs $2.40 \times 10^{-3}$ in SE negative cases) ) and of anti-CCP antibody ( $p=1.50 \times 10^{-11}$ in anti-CCP positive vs $5.10 \times 10^{-2}$ in anti-CCP negative cases)) on this association. Thus, the PTPN22 risk allele is associated with SE positivity in an RA population in which NKAPL effects on risk are primarily observed in SE negative disease.

Table 3 Associations of the NKAPL locus rs13208096 and rs13708096 SNPs with rheumatoid arthritis in the combined Canadian and US cohort

\begin{tabular}{|c|c|c|c|c|c|c|c|c|}
\hline \multirow[b]{2}{*}{ SNP } & \multirow[b]{2}{*}{ Gene } & \multirow[b]{2}{*}{ Risk allele } & \multirow[b]{2}{*}{ Sample cohort } & \multicolumn{2}{|l|}{ RAF } & \multirow[b]{2}{*}{ OR (95\% Cl) } & \multirow[b]{2}{*}{$\mathbf{P}_{\text {Comb }}$} & \multirow[b]{2}{*}{ Meta $0 \mathrm{p}$ value } \\
\hline & & & & Case & Control & & & \\
\hline rs35656932 & ZNF193 & $\mathrm{T}$ & $\begin{array}{l}\text { Toronto } \\
\text { Halifax } \\
\text { USA } \\
\text { Combined* }\end{array}$ & $\begin{array}{l}0.118 \\
0.119 \\
0.088 \\
0.109\end{array}$ & $\begin{array}{l}0.075 \\
0.083 \\
0.070 \\
0.074\end{array}$ & $\begin{array}{l}1.66(1.38 \text { to } 1.99) \\
1.48(1.11 \text { to } 1.99) \\
1.29(1.02 \text { to } 1.62) \\
1.50(1.32 \text { to } 1.71)\end{array}$ & $\begin{array}{l}3.82 \times 10^{-8} \\
8.31 \times 10^{-3} \\
0.032 \\
4.24 \times 10^{-10}\end{array}$ & 0.23 \\
\hline rs13208096 & NKAPL & G & $\begin{array}{l}\text { Toronto } \\
\text { Halifax } \\
\text { USA } \\
\text { Combined }\end{array}$ & $\begin{array}{l}0.115 \\
0.116 \\
0.086 \\
0.107\end{array}$ & $\begin{array}{l}0.075 \\
0.081 \\
0.068 \\
0.073\end{array}$ & $\begin{array}{l}1.61(1.34 \text { to } 1.94) \\
1.48(1.10 \text { to } 1.99) \\
1.30(1.03 \text { to } 1.63) \\
1.48(1.32 \text { to } 1.69)\end{array}$ & $\begin{array}{l}3.69 \times 10^{-7} \\
8.75 \times 10^{-3} \\
0.028 \\
2.44 \times 10^{-9}\end{array}$ & 0.35 \\
\hline
\end{tabular}

${ }^{*}$ Combined $p$ values for association $\left(P_{\text {Comb }}\right)$ were performed using Cochran-Mantel Haenszel tests stratifying by centre. The Cochran 0 tests for heterogeneity in the effect among studies.

NKAPL, NF-KB activating protein-like; RAF: risk allele frequency. 
Table 4 Analysis of NKAPL and PTPN22 risk allele disease associations in rheumatoid arthritis patient subgroups

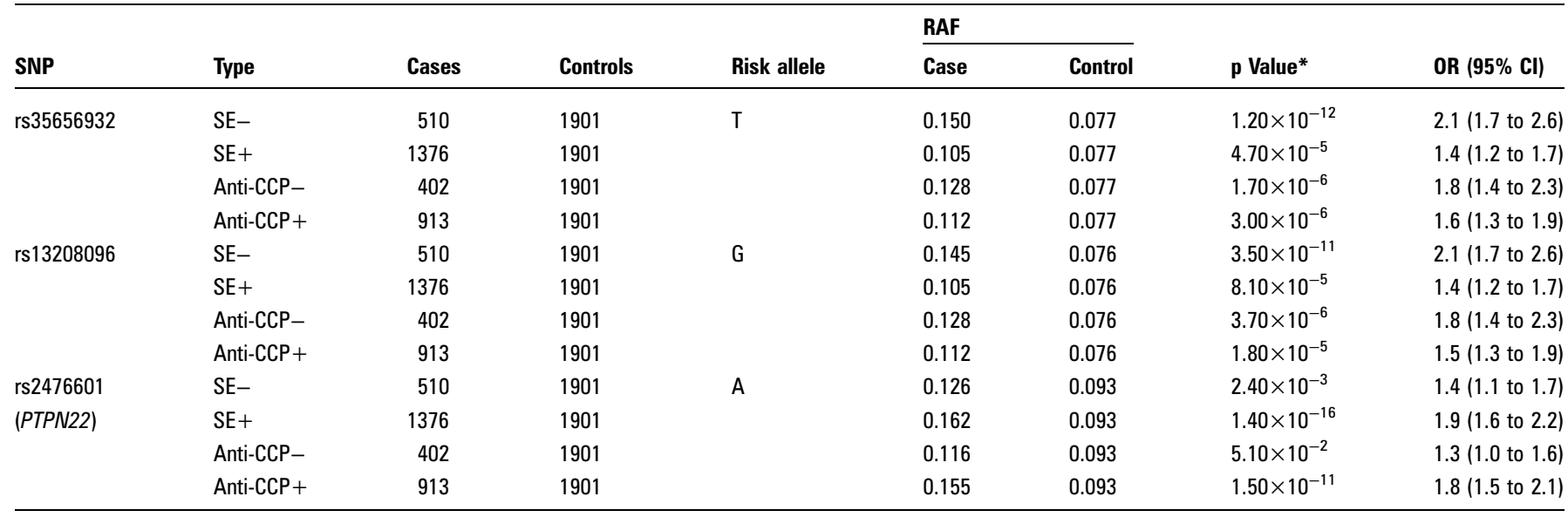

Data for subphenotype analyses were available for 1886 Canadian cases.

*Versus healthy controls.

CCP, cyclic citrullinated peptide; NKAPL, NF-kB activating protein-like; RAF: risk allele frequency; SE, presence of shared epitope encoding $D R B 1{ }^{*} 0101,{ }^{*} 0102,{ }^{*} 0401,{ }^{*} 0404$,

${ }^{*} 0405,{ }^{*} 0408,{ }^{*} 0409,{ }^{*} 1001$ or ${ }^{*} 1402$ alleles.

Effects of the NKAPL locus on risk were the strongest in the SE negative patients and increased when conditioning on HLA-DRB1 *0401 tag SNPs, suggesting interaction between NKAPL and the HLA-DRB1 risk alleles. This possibility was directly examined using case-only logistic regression models wherein SE positivity was considered the outcome and NKAPL genotype an additive effect. This analysis (table 5) revealed significant interactions between risks conferred by HLA-DRB1 alleles and the NKAPL rs35656932 and rs13208096 SNPs, with the interaction effect being negative for both the latter SNPs $(\mathrm{OR}=0.67 / \mathrm{CI} 0.54$ to 0.83 and $\mathrm{OR}=0.69 / \mathrm{CI} 0.55$ to 0.86 , respectively). By comparison, a positive interaction effect was apparent between the RA-associated PTPN22 rs2476601 variant and $\mathrm{SE}$ positivity $(\mathrm{OR}=1.34$, CI 1.08 to 1.65$)$. Results of multinomial logistic regression analyses (see online supplementary table S7) further confirmed strong interaction of the HLA-DRB1 SE alleles with each of the NKAPL $\left(p=4.35 \times 10^{-10}\right.$ and $\left.2.51 \times 10^{-11}\right)$ and the PTPN22 $\left(p=2.89 \times 10^{-15}\right)$ risk alleles and again revealed the ORs associated with either of the two NKAPL risk alleles to be lower, but for the PTPN22 risk allele to be higher, in a comparison of SE positive cases to controls versus SE negative cases to controls.

\section{DISCUSSION}

The NKAPL region emerged as a candidate RA risk locus in the context of our prior GWAS data providing strong evidence for association of SNPs across this locus with RA in a Canadian and US study population. Because the association was supported in subsequent meta-analysis incorporating this and four more GWAS datasets, ${ }^{10}$ we undertook fine mapping and

Table 5 HLA-DRB1 interaction with NKAPL or PTPN22 risk alleles

\begin{tabular}{llll}
\hline SNP & $\chi^{2}$ & p Value & OR (95\% Cl) \\
\hline rs35656932 & 13.44 & $2.0 \times 10^{-4}$ & $0.67(0.54$ to 0.83$)$ \\
rs13208096 & 11.27 & $8.0 \times 10^{-4}$ & $0.69(0.55$ to 0.86$)$ \\
rs2476601 & 7.15 & $7.5 \times 10^{-3}$ & $1.34(1.08$ to 1.65$)$
\end{tabular}

Logistic regression analysis using case-only design was used to test for interactions between HLA-DRB1 SE alleles and the NKAPL rs35656932 and rs13208096 SNPs or the PTPN22 rs2476601 risk variant.

NKAPL, NF- $\mathrm{KB}$ activating protein-like. conditional analyses to screen for risk variants with stronger and/or independent signals of disease association. By genotyping our Canadian RA case/control cohort for 101 SNPs across the locus, we have identified six variants for which association with RA reaches a conservative level of genome-wide significance $\left(p<5.7 \times 10^{-8}\right)$. Haplotype analysis reveals these six variants to all lie within one of these haplotype blocks in a region encompassing the NKAPL and three zing finger transcription factor genes. These genes are in strong LD with one another, but results of stepwise and conditional logistic regression analyses indicate that both ZNF193 rs35656932 and NKAPL rs13208096 SNPs contribute to risk for RA, associations for these two markers remaining significant after conditioning for each other associated SNP and results of stepwise logistic regression also suggesting independent effects of these SNPs on risk. These two associations were also replicated, albeit at modest levels of significance, in an independent US-based cohort.

Interpreting effects of the NKAPL locus on RA risk is complicated by the location of this locus in a chromosomal region (6p22.1) upstream of the HLA class II genes. While the NKAPL locus maps about $4306 \mathrm{~kb}$ away from the HLA-DRB1 gene and $1386 \mathrm{~kb}$ upstream from the telomeric end of the HLA region, the extensive $\mathrm{LD}$ across the region raises the possibility that the NKAPL association signals reflect LD with HLA-DRB1 SE alleles. However, effects of NKAPL as well as DRB1 SNPs on disease risk were revealed here by the logistic regression analyses conditioning on either of two HLA-DRB1*0401 tag SNPs. Analysis of pairwise LD between each of the most strongly associated NKAPL SNPs and the HLA-DRB1 tag SNPs also provided no evidence for LD $\left(\mathrm{r}^{2}<0.01\right)$ between SNPs at these respective loci and a stepwise logistic regression analysis combining the top six candidate SNPs from the fine-mapping study, 379 HLA region SNPs from the GWAS and the imputed HLA alleles further support contribution of the NKAPL locus to risk for RA. These findings are consistent with other data suggesting that the MHC locus contains loci in addition to HLA-DRB1 that confer risk to RA. ${ }^{28}$ Importantly, primary association of this locus with SE negative disease also implies interaction between the NKAPL and $H L A-D R B 1$ risk alleles, a possibility supported by the results of case-only and multinomial logistic analyses showing very significant negative interaction effects 
between HLA-DRB1 and each of two NKAPL risk alleles. While both the NKAPL and the observed PTPN22-HLA-DRB1 interaction effects on risk require further evaluation in relation to their biological significance, these data provide new insights into the complex effector interactions that may link risk genotypes to disease pathogenesis in RA.

The current data identify two SNPs, rs13208096 and rs35656932, as the major drivers of the association signal at the NKAPL locus. Among these, rs13208096 maps 1787bp upstream of NKAPL gene expressed in many tissues, including most immune cell populations. ${ }^{29}$ The gene encodes a 402 amino acid nuclear protein highly homologous to the NKAP that functions as a transcriptional repressor of NOTCH signalling in thymocytes and is required for haemopoietic stem cell maintenance and survival. ${ }^{30} 31$ NKAPL is not functionally characterised, but shares with NKAP a domain critical to NKAP roles in transcriptional repression ${ }^{30}$ and recent data from genome-wide annotation of transcriptional regulators (ENCONE:UCSC genome browser: http://genome.ucsc.edu/) reveal the rs13208096 SNP to be located in a region containing putative transcriptional regulatory histone marks.

The second SNP that drives the association signal at the NKAPL locus, rs35656932, maps 1741 bp upstream of the ZNF193 gene encoding the zinc finger transcription protein, ZNF193. Little is known about the functions of ZNF193 or the ZNF187 and ZNF307 proteins encoded by the nearby genes. However, ZNF307 has transcriptional repressor activity and appears to target $N F-\kappa B$, raising the intriguing, albeit highly speculative possibility that several genes at the NKAPL locus influence RA risk via effects on $N F-\kappa B$ signalling. ${ }^{32} 33$

Thus, while the current data do not identify the diseasecausal allele at the NKAPL locus, our findings provide compelling evidence that this locus confers risk for RA and that the variants accounting for this association signal emanate from highly plausible candidate genes likely to influence $N F-\kappa B$ as well as HLA-DRB1-modulated immune cell responses already implicated in RA risk and pathogenesis.

Acknowledgements This work was supported by grants from the Canadian Institutes for Health Research (MOP74621) and Ontario Research Fund (RE-01-061). KAS holds the Sherman Family Chair in Genomic Medicine and a Tier 1 Canada Research Chair. PAG, CIA and YL were partially supported by US NIH Grant AR44422.

Contributors GX planned and performed experiments, aided in writing the paper. Yue Lu and YS carried out statistical work, aided in preparing table. SSZ carried out DNA preparation and PCR reactions. ECK and RMP provided patient samples and data, aided in writing the paper. PKG provided data, aided in writing the paper. CIA planned the paper, guided statistical analyses, aided in data interpretation and paper writing. KAS led the planning and performance of experiments, aided interpretation of data and writing of the paper.

Funding None.

Competing interests None.

Patient consent Obtained.

Ethics approval Mount Sinai Hospital Institutional Review Board.

Provenance and peer review Not commissioned; externally peer reviewed.

Open Access This is an Open Access article distributed in accordance with the Creative Commons Attribution Non Commercial (CC BY-NC 3.0) license, which permits others to distribute, remix, adapt, build upon this work non-commercially, and license their derivative works on different terms, provided the original work is properly cited and the use is non-commercial. See: http://creativecommons.org/ licenses/by-nc/3.0/

\section{REFERENCES}

1. Firestein GS. Evolving concepts of rheumatoid arthritis. Nature 2003:423:356-61.

2. Plenge RM, Seielstad M, Padyukov L, et al. TRAF1-C5 as a risk locus for rheumatoid arthritis-a genomewide study. N Engl J Med 2007;357:1199-209.
3. Remmers EF, Plenge RM, Lee AT, et al. STAT4 and the risk of rheumatoid arthritis and systemic lupus erythematosus. N Engl J Med 2007;357:977-86.

4. Plenge RM, Cotsapas C, Davies L, et al. Two independent alleles at 6q23 associated with risk of rheumatoid arthritis. Nat Genet 2007;39:1477-82.

5. Thomson W, Barton A, Ke X, et al. Rheumatoid arthritis association at 6q23. Nat Genet 2007;39:1431-3

6. Barton A, Thomson W, Ke X, et al. Rheumatoid arthritis susceptibility loci at chromosomes 10p15, 12q13 and 22q13. Nat Genet 2008;40:1156-9.

7. Raychaudhuri S, Remmers EF, Lee AT, et al. Common variants at CD40 and other loci confer risk of rheumatoid arthritis. Nat Genet 2008:40:1216-23.

8. Raychaudhuri S, Thomson BP, Remmers EF, et al. Genetic variants at CD28, PRDM1 and CD2/CD58 are associated with rheumatoid arthritis risk. Nat Genet 2009;41:1313-18.

9. Gregersen PK, Amos Cl, Lee AT, et al. REL, encoding a member of the NF-kappaB family of transcription factors, is a newly defined risk locus for rheumatoid arthritis. Nat Genet 2009;41:820-3.

10. Stahl EA, Raychaudhuri S, Remmers EF, et al. Genome-wide association study meta-analysis identifies seven new rheumatoid arthritis risk loci. Nat Genet 2010;42:508-14.

11. Chen D, Li Z, Yang 0 , et al. Identification of a nuclear protein that promotes NF-kappaB activation. Biochem Biophys Res Commun 2003;310:720-4.

12. Plenge RM. Recent progress in rheumatoid arthritis genetics: one step towards improved patient care. Curr Opin Rheumatol 2009;21:262-71.

13. Gregersen PK, Olsson LM. Recent advances in the genetics of autoimmune disease. Annu Rev Immunol 2009;27:363-91.

14. Begovich AB, Carlton VE, Honigberg LA, et al. A missense single-nucleotide polymorphism in a gene encoding a protein tyrosine phosphatase (PTPN22) is associated with rheumatoid arthritis. Am J Hum Genet 2004;75:330-7.

15. Miretti MM, Walsh EC, Ke X, et al. A high-resolution linkage-disequilibrium map of the human major histocompatibility complex and first generation of tag single-nucleotide polymorphisms. Am J Hum Genet 2005;76:634-46.

16. Gabriel SB, Schaffner SF, Nguyen $\mathrm{H}$, et al. The structure of haplotype blocks in the human genome. Science 2002;296:2225-9.

17. Kazma R, Babron MC, Génin E. Genetic association and gene-environment interaction: a new method for overcoming the lack of exposure information in controls. Am J Epidemiol 2011;173:225-35.

18. Jawaheer $\mathbf{D}$, Seldin $\mathrm{MF}, \mathrm{Amos} \mathrm{Cl}$, et al. Screening the genome for rheumatoid arthritis susceptibility genes: a replication study and combined analysis of 512 multicase families. Arthritis Rheum. 2003:48:906-16.

19. Cornélis F, Fauré $S$, Martinez $M$, et al. New susceptibility locus for rheumatoid arthritis suggested by a genome-wide linkage study. Proc Natl Acad Sci USA 1998;95:10746-50

20. MacKay K, Eyre S, Myerscough A, et al. Whole-genome linkage analysis of rheumatoid arthritis susceptibility loci in 252 affected sibling pairs in the United Kingdom. Arthritis Rheum 2002;46:632-9.

21. Gregersen PK, Silver J, Winchester RJ. The shared epitope hypothesis. An approach to understanding the molecular genetics of susceptibility to rheumatoid arthritis. Arthritis Rheum 1987;30:1205-13.

22. Stenzel A, Lu T, Koch WA, et al. Patterns of linkage disequilibrium in the MHC region on human chromosome 6p. Hum Genet 2004;114:377-85.

23. Newton JL, Harney SM, Wordsworth BP, et al. A review of the MHC genetics of rheumatoid arthritis. Genes Immun 2004:5:151-7.

24. Irigoyen $\mathbf{P}$, Lee AT, Wener MH, et al. Regulation of anti-cyclic citrullinated peptide antibodies in rheumatoid arthritis: contrasting effects of HLA-DR3 and the shared epitope alleles. Arthritis Rheum 2005;52:3813-8.

25. Huizinga TW, Amos $\mathrm{Cl}$, van der Helm-van Mil AH, et al. Refining the complex rheumatoid arthritis phenotype based on specificity of the HLA-DRB1 shared epitope for antibodies to citrullinated proteins. Arthritis Rheum 2005:52:3433-8.

26. Orozco G, Pascual-Salcedo D, López-Nevot MA, et al. Auto-antibodies, HLA and PTPN22: susceptibility markers for rheumatoid arthritis. Rheumatology (Oxford) 2008;47:138-41.

27. Mahdi H, Fisher BA, Källberg $H$, et al. Specific interaction between genotype, smoking and autoimmunity to citrullinated alpha-enolase in the etiology of rheumatoid arthritis. Nat Genet 2009;41:1319-24.

28. Lee HS, Lee AT, Criswell LA, et al. Several regions in the major histocompatibility complex confer risk for anti-CCP-antibody positive rheumatoid arthritis, independent of the DRB1 locus. Mol Med 2008;14:293-300.

29. Su Al, Wiltshire T, Batalov $S$, et al. A gene atlas of the mouse and human protein-encoding transcriptomes. Proc Natl Acad Sci USA 2004:101:6062-7.

30. Pajerowski AG, Nguyen C, Aghajanian $\mathrm{H}$, et al. NKAP is a transcriptional repressor of notch signaling and is required for T cell development. Immunity 2009:30:696-707.

31. Pajerowski AG, Shapiro MJ, Gwin K, et al. Adult hematopoietic stem cells require NKAP for maintenance and survival. Blood 2010;116:2684-93.

32. Laity $\mathbf{J H}$, Lee BM, Wright PE. Zinc finger proteins: new insights into structural and functional diversity. Curr Opin Struct Biol 2001:11:39-46.

33. Li J, Wang Y, Fan $\mathrm{X}$, et al. ZNF307, a novel zinc finger gene suppresses $p 53$ and p21 pathway. Biochem Biophys Res Commun 2007;363:895-900. 